Gastroenterologe 2009 · 4:391-392

DOI 10.1007/s11377-008-0234-6

(c) Springer Medizin Verlag 2009

\author{
M.W. Büchler ${ }^{1}$ J.F. Riemann ${ }^{2}$ \\ ${ }^{1}$ Chirurgische Klinik, Universitätsklinikum Heidelberg \\ ${ }^{2}$ Ludwigshafen
}

\title{
Viszeralmedizin
}

\section{Komplexe Krankheitsbilder aus gastroenterologischer und chirurgischer Sicht}

Gastroenterologie und Viszeralchirurgie treffen sich täglich am Patienten bzw. in den regelmäßig stattfindenden Boards, wo wir über die im Einzelfall so wichtige Frage einer nichtoperativen oder chirurgischen Behandlung diskutieren und entscheiden. Von ganz besonderer Bedeutung sind hierbei Krankheitsbilder, bei denen in der Tat im Einzelfall eine nichtchirurgische/endoskopische/konservative Therapie zum Ziel führen, im anderen Falle jedoch die chirurgische Entfernung bzw. Behandlung evidenzbasiert überlegen sein kann. Die aktuelle Entwicklung in unseren Krankenhäusern hin zu einer Viszeralmedizin ist daher wegweisend.

Das vorliegende Heft der Zeitschrift „Der Gastroenterologe“ greift sechs Grenzbereiche zwischen Gastroenterologie und Viszeralchirurgie auf, und wir freuen uns sehr, dass es den Autoren gelungen ist, im jeweiligen Artikel gemeinsam die Schnittmengen, aber sicherlich auch die Trennlinien zwischen konservativer und operativer Medizin zu definieren.

Die gastroösophageale Refluxkrankheit ist ohne Zweifel eine Domäne der konservativen Therapie. Nur bei ganz spezieller Indikation, nämlich bei Therapieresistenz bzw. bei besonderen anatomischen Formen der Hiatushernien, kommt der Chirurg mit der heute vorzugsweise anzuwendenden laparoskopischen Fundoplikation zum Zug. Weitere Indikationen zur seltenen chirurgischen Therapie sind die unzureichende Compliance des Patienten und der Mischreflux bzw. der voluminöse Reflux, der durch antisekretorische Therapie nicht hinreichend behandelt werden kann. Die Nebenwirkungen der chirurgischen Therapie, wie Dysphagie oder
Gas-Bloat-Syndrom, müssen dem Patienten präoperativ kommuniziert werden.

Der gutartige Tumor der Ampulla Vateri ist heute eine Domäne der endoskopischen Therapie. An diesem Beispiel kann eindrucksvoll die Entwicklung moderner endoskopischer Resektionsverfahren dargestellt werden. Die Trennlinie zwischen endoskopischer und chirurgischer Therapie ist das voll entwickelte Karzinom an der Papille, weil hier die radikale chirurgische Therapie mit 5-Jahres-Überlebensraten von über $50 \%$ positiv zum Zug kommt. Die intensive interdisziplinäre Diskussion zwischen dem endoskopisch aktiven Gastroenterologen und dem Viszeralchirurgen bei Übergangsformen zwischen Adenomen und Karzinomen erbringt heute die beste Therapie für diese Patienten. In der Regel wird man die hochgradig dysplastischen Adenome endoskopisch abtragen und bei freien Resektionsrändern einem Follow-up zuführen, bei einem Karzinombefund im endgültigen Resektat jedoch eine radikale Nachresektion befürworten.

Die nekrotisierende Pankreatitis hat wahrscheinlich wie kaum eine andere gastroenterologische Erkrankung in den vergangenen 30 Jahren ein Wechselspiel des Therapiezuganges erlebt. Während in den 8oer Jahren des vergangenen Jahrhunderts noch großzügig die Indikation zur Operation gestellt wurde, ist heute die nichtchirurgische konservative Therapie dieser lebensbedrohlichen Erkrankung mit wenigen Ausnahmen Standard der Therapie. Was heute mit dem Schlagwort „NOTES“ unsere gastroenterologische und chirurgische und viszeralchirurgische Weltanschauung beeinflusst, wurde bei der ne- krotisierenden Pankreatitis bereits pionierhaft in den goer Jahren durch Seifert et al. [1] umgesetzt; diese Gruppe hat den transgastrischen Zugang zur Pankreasnekrose und deren Ausräumung mit endoskopischen Maßnahmen entwickelt.

Ein weiteres Paradebeispiel der interdisziplinären Abstimmung zwischen Gastroenterologen und Viszeralchirurgen ist die Frage der Lebertransplantation bei primär sklerosierender Cholangitis (PSC). Obwohl das Risiko einer Karzinomentwicklung bei bestehender PSC bis zu $20 \%$ erhöht ist, rechtfertigt dies jedoch alleine noch nicht die prophylaktische Lebertransplantation, sondern es muss individuell in der Betrachtung eines Krankheitsverlaufes und in der argumentativen Abstimmung zwischen Internist und Chirurg entschieden werden. Durch gastroenterologische und insbesondere endoskopische Techniken gelingt es heute, die PSC in vielen Fällen langfristig zu kontrollieren und natürlich auch die PSC-assoziierte Colitis ulcerosa.

Der fistelnde Morbus Crohn, insbesondere wenn er den Anus befällt, ist eine weitere Domäne der interdisziplinären Therapie. Die chirurgische Therapie des Morbus Crohn tritt heute Dank effektiver Medikation in den Hintergrund. Der fortgeschrittene Morbus Crohn am Anus ist aber nach wie vor ein ungelöstes Problem; hier müssen möglichst kleine chirurgische Eingriffe mit aktiver medikamentöser Therapie kombiniert angewandt werden.

Chirurgen machen gerne große Operationen. Der Langzeitverlauf nach kompletter Entfernung des Magens oder der Bauchspeicheldrüse wird jedoch beeinträchtigt von den unterschiedlichsten 
Mangelerscheinungen der Patienten. Gelingt es, die Tumorerkrankung zu heilen, dann ist die Angelegenheit für den Patienten nach solch ausgedehnten Operationen bei weitem nicht erledigt. Die postoperativen Probleme nach Magen- und Pankreasresektion sind daher ein weiteres Beispiel für die so notwendige Abstimmung zwischen Gastroenterologen und Viszeralchirurgen.

Die Herausgeber des vorliegenden Heftes freuen sich über die gelungene Abbildung der Schnittmengen zwischen Gastroenterologie und Viszeralchirurgie anhand von sechs Beispielen. Die Manuskripte wurden von ausgewiesenen Experten erstellt, und wir sind außerordentlich dankbar dafür. Wir dürfen der Leserschaft von „Der Gastroenterologe“ diese wichtigen, im Konsens erstrittenen Ergebnisse der beispielhaften interdisziplinären Behandlung unserer Patienten ans Herz legen.

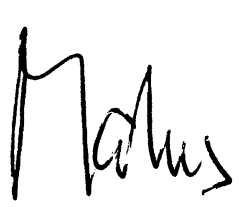

M.W. Büchler

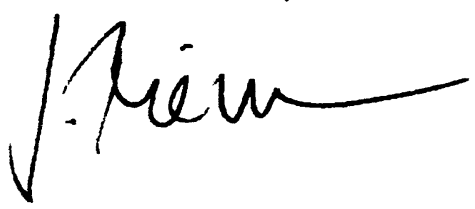

J.F. Riemann

\section{Korrespondenzadressen}

Prof. Dr. M.W. Büchler

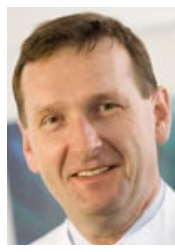

Chirurgische Klinik,

Universitätsklinikum Heidelberg

Im Neuenheimer Feld

110, 69120 Heidelberg

markus_buechler@med.

uni-heidelberg.de

\section{Prof. Dr. J.F. Riemann}

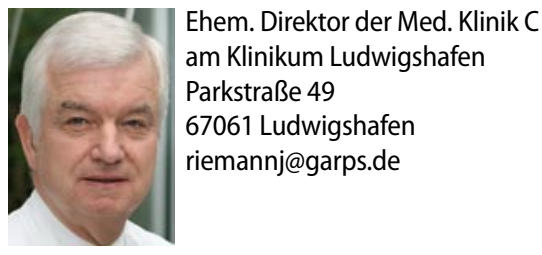

\section{Literatur}

1. Seifert H, Wehrmann T, Schmitt T, Zeuzem S, Caspary WF (2000) Retroperitoneal endoscopic debridement for infected peripancreatic necrosis. Lancet 356: 653-655
Der Gastroenterologe bietet umfassende und aktuelle Beiträge zu interessanten Themenschwerpunkten aus allen Bereichen der Gastroenterologie und Hepatologie.

Möchten Sie ein bereits erschienenes Heft nachbestellen? Die folgenden Ausgaben aus 2008 und bereits erschienene Ausgaben aus 2009 können Sie direkt bei unserem Kundenservice zum Preis von EUR 32,- beziehen:

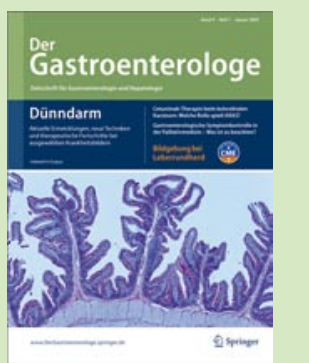

\section{8}

- 1/08 Gallenwegserkrankungen

- 2/08 Kolonkarzinom - Update

- 3/08 Hereditäre Lebererkrankungen

- 4/08 Reflux und refluxassoziierte Erkrankungen

- 5/08 Adipositas

- 6/08 Neurogastroenterologie/Motilität

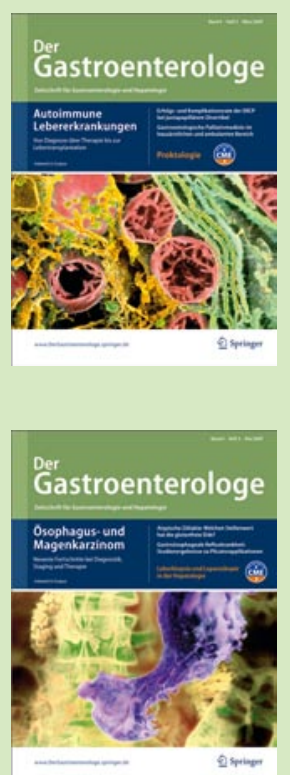

\section{9}

- 1/09 Dünndarm

- 2/09 Autoimmune Lebererkrankungen

- 3/09 Ösophagus- und Magenkarzinom

- 4/09 Viszeralmedizin - komplexe Krankheitsbilder aus hepatologischer und chirurgischer Sicht

- 5/09 Viszeralmedizin - komplexe Krankheitsbilder aus gastroenterologischer und chirurgischer Sicht

6/09 Leber und Pankreas

(Änderungen vorbehalten)

So erreichen Sie unseren Kundenservice:

Springer Medizin Verlag GmbH

Kundenservice Zeitschriften

Haberstraße 7

69126 Heidelberg

Tel. $+496221345-4303$

Fax: +49 6221 345-4229

E-Mail: subscriptions@springer.com

www.DerGastroenterologe.springer.de 